\title{
Impacto del ejercicio sobre el metabolismo de los lípidos y la dislipidemia
}

\author{
Impact of exercise on lipid metabolism and dyslipidemia \\ Impacto do exercício sobre o metabolismo dos lipídeos e da dislipidemia
}

Eitan A. Scher-Nemirovsky' ${ }^{1}$ Daniel Ruiz-Manco', Carlos O. Mendivil ${ }^{1,2^{*}}$

Recibido: 16 de julio de 2019. Aceptado para publicación: 5 de agosto de 2019

https://doi.org/10.35454/rncm.v2n2.004

\section{Resumen}

La práctica crónica del ejercicio induce una serie de adaptaciones celulares y orgánicas que modifican la forma en que el cuerpo humano metaboliza todos los macronutrientes, incluidos los lípidos. El ejercicio de duración y el ejercicio de resistencia provocan diferentes respuestas que resultan en efectos diferenciales sobre el metabolismo de los lípidos y las lipoproteínas. Estos efectos son cuantitativa y cualitativamente diferentes y mediados por distintas vías de señalización. Esta revisión, resume la evidencia pertinente sobre la repercusión del ejercicio en el metabolismo de los lípidos y las lipoproteínas, y finaliza con algunas recomendaciones sobre la práctica del ejercicio para los pacientes con dislipidemia en el ámbito de la atención primaria.

Palabras clave: lípidos, actividad física, atención primaria.

\section{Summary}

The chronic practice of exercise induces a series of cellular and organismal adaptations that modify the way the human body metabolizes all macronutrients, including lipids. Endurance exercise and resistance exercise elicit different responses that result in differential effects on lipid and lipoprotein metabolism. These effects are quantitatively and qualitatively different and mediated by distinct signaling pathways. In this review, we summarize relevant evidence on the impact of exercise on lipid and lipoprotein metabolism, and finalize with some practical recommendations on exercise practice for patients with dyslipidemia in the primary care setting.

Keywords: Lipids; Physical activity; Primary attention.

\section{Resumo}

A prática crônica de exercício induz uma série de adaptações celulares e orgânicas que modificam a maneira pela qual o corpo humano metaboliza todos os macronutrientes, incluindo os lipídios. O exercício de duração e o exercício de resistência provocam diversas respostas que resultam em efeitos diferenciais no metabolismo de lipídios e lipoproteínas. Estes efeitos são quantitativa e qualitativamente distintos e mediados por diferentes vias de sinalização. Nesta revisão, se resume as evidências relevantes sobre o impacto do exercício no metabolismo de lipídios e das lipoproteínas e conclui, com algumas recomendações sobre a prática de exercícios para pacientes com dislipidemia no campo da atenção primária.

Palavras-chave: lipídios, atividade física, atenção primária.

\section{INTRODUCCIÓN}

Durante la evolución, solo los más aptos lograron sobrevivir y transmitir sus genes a la siguiente generación. En una época en la que los recursos eran escasos, los humanos más aptos eran aquellos que podían sopor-

\footnotetext{
Facultad de Medicina, Universidad de los Andes, Bogotá, D.C., Colombia

Sección de Endocrinología, Fundación Santa Fe de Bogotá, Bogotá, D.C., Colombia.
}

tar largas horas de caza o de trabajo. Esto significa que los genes que codifican el almacenamiento eficiente de energía y el metabolismo son favorecidos y transmitidos generación tras generación hasta ahora. Sin embargo, en los tiempos modernos de abundancia de recursos y producción mecanizada, la sobreabundancia de energía y el

Correspondencia: *Carlos O. Mendivil, MD, PhD. cmendivi@uniandes.edu.co 
bajo gasto son la norma. Esta inactividad física aumenta la incidencia de al menos 17 afecciones no saludables y enfermedades crónicas relacionadas, mientras que una baja capacidad de ejercicio es un indicador independiente de la mortalidad y morbilidad por todas las causas $^{(1)}$. Uno de los efectos demostrados del comportamiento sedentario es la disfunción metabólica, caracterizada por el aumento de los niveles de triglicéridos en plasma, la disminución de los niveles de colesterol de lipoproteínas de alta densidad (HDL) y la disminución de la sensibilidad a la insulina ${ }^{(2)}$. Todas estas consecuencias indeseables de la inactividad física justifican una comprensión de las vías fisiológicas por las que la actividad física confiere sus beneficios metabólicos.

\section{ACTIVIDAD FÍSICA, EJERCICIO Y DEPORTE}

Mientras la actividad física (AF) se refiere a cualquier tipo de movimiento corporal producido por la contracción consciente del músculo esquelético que exige el uso de energía ${ }^{(3)}$, el ejercicio es un tipo de actividad física planificada, estructurada, repetitiva y con propósito, en el sentido de que el objetivo es la mejora o el mantenimiento de uno o más componentes de la aptitud física ${ }^{(4)}$. Si bien es cierto que cada vez que la persona hace ejercicio hace $\mathrm{AF}$, no significa que cada vez que está físicamente activa esté haciendo ejercicio. Por último, deporte significa todas las formas de AF que, a través de la participación casual u organizada, tienen como objetivo expresar o mejorar la forma física y el bienestar mental, obteniendo resultados en la competición a todos los niveles, y estableciendo relaciones sociales.

La capacidad de coordinación y adaptación de una amplia gama de cualidades fisiológicas y cognitivas determina la forma física. Este término se divide a su vez en tres componentes que pueden ser mejorados a través de la formación continua, y son: la aptitud cardiorrespiratoria, la aptitud muscular y la velocidad/coordinación. $\mathrm{El}$ acondicionamiento cardiorrespiratorio es la capacidad general de los sistemas cardiovascular y respiratorio para realizar ejercicios vigorosos y prolongados. El American College of Sports Medicine define la capacidad aeróbica como el producto de la capacidad del sistema cardiorrespiratorio para suministrar oxígeno y la de los músculos esqueléticos para utilizarlo ${ }^{(5)}$. El consumo máximo de oxígeno $\left(\dot{\mathrm{V}}_{2 \max }\right)$ alcanzado durante un ejercicio graduado hasta el agotamiento voluntario ha sido considerado, desde hace tiempo, como el mejor indicador del estado físico cardiorrespiratorio. La aptitud muscular es la capacidad de realizar un trabajo contra una resistencia. La fuerza máxima que se puede generar depende de varios factores, incluyendo el tamaño y el número de músculos involucrados, la proporción de fibras musculares llamadas a la acción y la coordinación de los grupos musculares.

Los principales componentes de la aptitud muscular relacionados con la salud son la fuerza máxima (isométrica y dinámica), la fuerza explosiva, la fuerza de resistencia y la fuerza isocinética ${ }^{(6)}$. La velocidad es la capacidad de mover el cuerpo (o algunas partes del cuerpo) lo más rápido posible. La agilidad es la capacidad de moverse rápidamente y cambiar de dirección mientras se mantiene el control y el equilibrio. Por consiguiente, la agilidad es una combinación de velocidad, equilibrio, potencia y coordinación ${ }^{(6)}$.

\section{RESPUESTA FISIOLÓGICA A LA ACTIVIDAD FÍSICA}

Para apoyar las demandas constantes de la AF, el cuerpo responde con una adaptación multisistémica que mantiene el suministro de oxígeno y sustratos metabólicos al músculo esquelético. Las adaptaciones crónicas que optimizan la generación, oxidación y consumo de sustratos y cambios celulares serán discutidas más adelante. Las respuestas agudas al ejercicio son:

- Cardiovascular: el flujo sanguíneo al músculo esquelético activo puede aumentar 100 veces por encima delos niveles basales, representando hasta $80 \%$ - $90 \%$ del gasto cardiaco. El aumento gigantesco en el flujo sanguíneo del músculo esquelético se logra en gran medida por el aumento del gasto cardíaco por medio de la bomba del músculo esquelético que promueve el retorno venoso durante el ejercicio dinámico, pero también en parte desviando el flujo de los riñones y los órganos esplánicos. En particular, solo hay un modesto (20\%) aumento de la presión arterial media (PAM), mientras que los valores de $\mathrm{PO}_{2}$, $\mathrm{PCO}_{2}$ y $\mathrm{pH}$ arterial permanecen esencialmente idénticos al valor de reposo hasta que se alcanzan las intensidades máximas de ejercicio ${ }^{(1)}$. La estabilidad de la PAM se mantiene principalmente debido a la disminución de la resistencia periférica total causada por la acumulación de vasodilatadores metabólicos y la disminución de la resistencia vascular en el músculo esquelético activo, que es una alteración que reduce la presión y que provoca un fuerte aumento de la actividad simpática a través del reflejo arterial de barorreceptor. El ejercicio estático (es decir, iso- 
métrico) presenta una alteración muy diferente en el sistema cardiovascular que el ejercicio dinámico. Como se discutió en la sección anterior, el ejercicio dinámico produce grandes reducciones en la resistencia periférica total debido a la vasodilatación metabólica local en los músculos de ejercicio. Los esfuerzos estáticos, incluso de intensidad moderada, provocan una compresión de los vasos en los músculos que se contraen y una reducción del flujo sanguíneo a través de ellos.

Por lo tanto, la resistencia periférica total puede aumentar de manera significativa. Los efectos cardiovasculares del ejercicio estático incluyen aumento en la frecuencia cardíaca, el gasto cardíaco y la presión $\operatorname{arterial}^{(7)}$.

- Respiratorio: las funciones críticas del sistema pulmonar son mantener la oxigenación arterial y facilitar la eliminación del $\mathrm{CO}_{2}$ producido durante el metabolismo oxidativo aumentando la ventilación en proporción a la intensidad del ejercicio ${ }^{(8)}$.

- Músculo esquelético: el ATP no se almacena en grandes cantidades dentro del músculo esquelético. Debido a su limitada disponibilidad, el ATP se resintetiza a un ritmo que permite satisfacer las demandas metabólicas que se imponen a la célula. La fuente de sustrato más inmediata para la resintetización de ATP en el músculo esquelético es la fosfocreatina (PCr). $\mathrm{El}$ músculo esquelético tiene suficiente $\mathrm{PCr}$ para mantener la tasa máxima de rotación de ATP durante unos 7 - 10 s. El músculo esquelético también utiliza tanto grasa como carbohidratos como sustratos para la resintetización de ATP. Las tasas a las que los AGF y los carbohidratos pueden reponer el ATP muscular son significativamente más bajas que las de PCr o ADP. Sin embargo, la cantidad de ATP que pueden producir a partir de una sola molécula de cualquiera de ellos es significativamente mayor. El aumento de la duración del ejercicio se logra a expensas de la tasa de rotación del ATP. La menor tasa de rotación de ATP puede ser igualada por la fosforilación oxidativa que emplea una combinación de glucosa/glicógeno y ácidos grasos como sustratos ${ }^{(9)}$.

- Metabolismo lipídico: las fuentes de energía durante el ejercicio se conocen desde los años sesenta ${ }^{(10)}$. En cargas de trabajo inferiores a $30 \%$ del $\dot{V O}_{2 \text { máx }}$ la principal fuente de energía son los ácidos grasos, entre $40 \%$ y $65 \%$ de $\dot{\mathrm{VO}}{ }_{2 \operatorname{máx}}$ hay aproximadamente un equilibrio de 50:50 entre los carbohidratos y la oxidación de las grasas, y más allá de $70 \%$ de $\dot{\mathrm{V}} \mathrm{O}_{2 \max }$ hay un aumento exponencial de la oxidación de los car- bohidratos con una disminución concomitante de la oxidación de las grasas ${ }^{(9)}$. La contribución relativa de la oxidación de las grasas al gasto total de energía varía muy poco hasta niveles intermedios de intensidad de ejercicio $^{(11)}$. Sin embargo, a medida que la intensidad del ejercicio aumenta hasta $75 \%$ de $\dot{\mathrm{V}} \mathrm{O}_{2 \text { máx }}$, la utilización del sustrato cambia de forma notable, la tasa de oxidación total de la grasa corporal disminuye, mientras que la glucogenolisis muscular se convierte en la fuente primaria de energía (Figura 1).

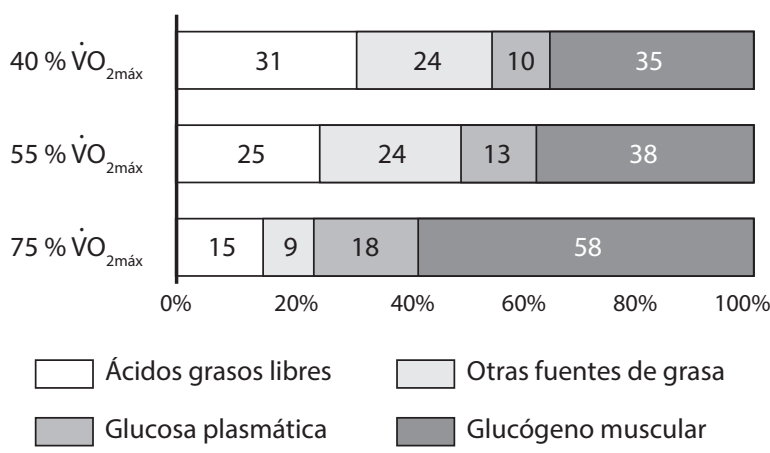

Figura 1. Porcentaje de energía total obtenida de cada fuente de energía según la intensidad del ejercicio. Datos de ${ }^{(11)}$.

\section{ADAPTACIÓN CRÓNICA AL EJERCICIO}

En los músculos humanos se conocen tres subgrupos de fibras: glicolítico de cambio rápido (FG), oxidativo de cambio rápido $(\mathrm{FO})$ y oxidativo de cambio lento $(\mathrm{SO})^{(12)}$. Las FG son de manera predominante fibras anaeróbicas, tienen una alta concentración de glucógeno y una alta actividad de fosforilasa, lactato deshidrogenasa y alfa-glicerofosfato deshidrogenasa. Las fibras FG tienen una rápida contracción y una tasa de explosividad, a expensas de una rápida fatigabilidad. Mientras tanto, las fibras de SO dependen en especial del metabolismo aeróbico, tienen una baja concentración de glucógeno y baja fosforilasa, deshidrogenasa láctica y actividades de la alfa-glicerofosfato deshidrogenasa mitocondrial. Las propiedades de las fibras de FO están a medio camino entre las de las fibras FG y $\mathrm{SO}$, con un alto índice de oxidación y glicogenolisis ${ }^{(13)}$.

$\mathrm{Al}$ considerar las adaptaciones fisiológicas crónicas al ejercicio, hay dos vías principales que activan respuestas muy distintas en la célula. Es importante tener en cuenta que cada vía es activada por diferentes tipos de entrenamiento. En el caso del entrenamiento de duración, favorece la vía que estimula la formación de nuevas mitocondrias, mejorando así el desarrollo de las fibras de SO. Por otro lado, el entrenamiento de resis- 
tencia favorece la vía hipertrófica mejorando el desarrollo de las fibras FG.

\section{Vía AMPK}

El entrenamiento aeróbico y de duración aumenta en forma considerable la relación AMP/ATP, lo que activa la vía AMPK. AMPK es un importante sensor de disminución de la carga energética en las células y, posteriormente, actúa para aumentar las reacciones catabólicas y disminuir las reacciones anabólicas, en particular para aumentar la absorción de glucosa a través de la expresión de GLUT4, aumentar la oxidación de los ácidos grasos y disminuir la síntesis de glucógeno ${ }^{(14)}$. La AMPK requiere el Coactivador del Receptor Activado por Proliferador del Peroxisoma gamma (PPARgamma) 1-alfa (PGC-1) para muchos de sus efectos sobre la expresión génica en el músculo esquelético. La AMPK se une y activa el PGC-1 en el músculo por fosforilación directa en dos residuos críticos, la treonina- 177 y la serina-538 $8^{(15)}$. El PGC-1 es un regulador crítico de la transcripción de muchos genes involucrados en la homeostasis de la energía, en particular la oxidación del combustible y la biología mitocondrial. Estas fosforilaciones mediadas por AMPK inducen al PGC-1alfa a activar una cascada transcripcional que termina en biogénesis mitocondrial y el ensamblaje estequiométrico de complejos proteicos multisubunitarios en una cadena respiratoria funcional. El factor nuclear respiratorio 1 y 2 , objetivos del PGC-1, regula directamente la expresión de los genes mitocondriales codificados y puede estimular la expresión del factor de transcripción mitocondrial A (Tfam), una proteína de la matriz mitocondrial esencial para la replicación y transcripción del ADN mitocondrial ${ }^{(16)}$.

\section{Vía mTOR}

El entrenamiento de resistencia activa una respuesta de señalización intracelular por completo diferente, provocada principalmente por la secreción de la hormona del crecimiento ${ }^{(17)}$. Se ha comprobado que varios protocolos de ejercicios de gran resistencia elevan la concentración de $\mathrm{GH}^{(17)}$ después del ejercicio. La GH regula procesos fisiológicos importantes, incluyendo el crecimiento y desarrollo somático, así como el metabolismo de los carbohidratos y lípidos, de manera directa a través de la activación de receptores específicos de GH, o de forma indirecta a través del factor de crecimiento similar a la insulina 1 (IGF$1)$, que se produce principalmente en el hígado en respuesta a la estimulación de $\mathrm{GH}^{(18)}$. La hipertrofia de miotubos in vitro inducida por IGF-1 dependía de una vía iniciada por la fosfoinositide 3-quinasa (PI3K) y la quinasa Akt regulada por PI3K, que a su vez condujo a la activación de la quinasa sensible a la rapamicina conocida como mTOR, cuyos objetivos descendentes, p70S6K y PHAS-1/4E-BP1, han demostrado promover la síntesis de proteínas a través de incrementos en la iniciación y alargamiento de la translación ${ }^{(19)}$. La fosforilación de la p70S6K (un efector descendente de mTOR) aumenta la fosforilación de la proteína ribosomal S6 y facilita la síntesis de algunas proteínas ribosomales, factores de iniciación y factores de elongación que desempeñan un papel importante en la síntesis de proteínas ${ }^{(20)}$. Un estudio in vivo en voluntarios humanos confirmó además este efecto: tras ocho semanas de entrenamiento de resistencia, la hipertrofia del músculo esquelético humano se asoció a un aumento de la cantidad de Akt fosforilado y $\mathrm{mTOR}^{(21)}$.

\section{HOMEOSTASIS DE LÍPIDOS Y LIPOPROTEÍNAS}

El término lípidos abarca un gran grupo de compuestos hidrofóbicos que no pueden viajar de forma eficiente en un solvente polar como el plasma humano. Para que puedan ser transportados a los diversos tejidos y órganos para su utilización y almacenamiento, los lípidos no polares (tricilglicerol y ésteres de colesterol) deben asociarse a los lípidos anfípticos (fosfolípidos y colesterol) y a las proteínas para obtener lipoproteínas miscibles en agua $^{(22)}$. Los lípidos plasmáticos comprenden los triacilgliceroles -también llamados triglicéridos- (16\%), fosfolípidos (30\%), colesterol (14\%), ésteres de colesterol (36\%) y una fracción mucho menor de ácidos grasos de cadena larga no esterificados (o ácidos grasos libres - AGL) $(4 \%)^{(23)}$.

Dado que la grasa es menos densa que el agua, la densidad de una lipoproteína disminuye a medida que aumenta la proporción de lípidos con respecto a las proteínas. Se han identificado cuatro grupos principales de lipoproteínas que son importantes desde el punto de vista fisiológico y clínico. Se trata de 1. quilomicrones, derivados de la absorción intestinal de tricilglicerol y otros lípidos; 2. VLDL, sintetizada por el hígado para la exportación de tricilglicerol; 3. lipoproteínas de baja densidad (LDL), que representan una etapa final en el catabolismo de la VLDL; y 4. lipoproteínas de alta densidad (HDL), que intervienen en el transporte de colesterol, así como en el transporte de VLDL y en el 
metabolismo de los quilomicrones ${ }^{(24)}$. Cada clase de lipoproteína contiene apolipoproteínas que definen su estructura y función. Las apolipoproteínas más importantes se resumen en la Tabla $1^{(22)}$.

\section{Metabolismo de quilomicrones}

Una vez absorbido en las células epiteliales intestinales, el TG de la dieta es secretado en el torrente sanguíneo dentro de los quilomicrones. El ensamblaje de quilomicrones requiere apoB-48 como apolipoproteína estructural. Una vez que los quilomicrones acceden al torrente sanguíneo, los triglicéridos en su núcleo son hidrolizados en ácidos grasos libres y glicerol por la lipoproteína lipasa-1 (LPL-1) (presente en la superficie de la mayoría de las células endoteliales) con la apolipoproteína C-II como cofactor. Este proceso produce restos de quilomicrones más pequeños ${ }^{(25)}$. Estos remanentes son eliminados posteriormente en especial por un receptor remanente de quilomicrones en el hígado, conocido como la proteína-1 del receptor similar a las LDL (LRP-1) ${ }^{(24)}$.

\section{Metabolismo de la VLDL}

La VLDL sufre un proceso bastante similar al de los quilomicrones, pero con diferentes actores. Las VLDL ricas en triglicéridos son sintetizadas y secretadas por el hígado, un proceso que requiere apoB - 100 en lugar de
apoB - 48. En el plasma, los triglicéridos de la VLDL se descomponen en ácidos grasos libres y glicerol por la lipoproteína lipasa con participación de apoC - II, tal como sucede con los quilomicrones. Esto resulta en la producción de remanentes más pequeños de VLDL e IDL (lipoproteínas de densidad intermedia). Algunas de las partículas de IDL se eliminan a través de la interacción de la apolipoproteína $\mathrm{E}$ con el receptor LDL en la superficie del hígado, mientras que otras pueden ser hidrolizadas aún más por la lipasa hepática para producir LDL y se elimina normalmente por la interacción de la apolipoproteína B-100 con el receptor LDL. ApoB comprende $23,8 \%$ del peso de la partícula LDL y es el determinante proteico para el reconocimiento celular y el catabolismo de $\mathrm{LDL}^{(26)}$. Cuando las LDL se oxidan, los macrófagos pueden fagocitarlas con más facilidad a través de los receptores CD36 y SR - $\mathrm{A}^{(24)}$.

\section{Metabolismo del HDL}

El HDL, sintetizado y secretado tanto por el hígado como por el intestino, son partículas complejas. Hasta la fecha, se han identificado más de 110 polipéptidos individuales en el HDL mediante diversos métodos ${ }^{(27)}$. Una de las principales funciones de HDL es actuar como repositorio de los apoC y apoE necesarios para el metabolismo de los quilomicrones y VLDL. El HDL es originalmente secretado como una partícula naciente rica en fosfolípidos, pobre en colesterol, que luego adquiere fosfolípidos

Tabla 1. Principales apolipoproteínas, sus funciones y localización.

\begin{tabular}{|c|c|c|c|}
\hline $\begin{array}{c}\text { Tipo de } \\
\text { apolipoproteína }\end{array}$ & Apolipoproteína & Función & Presente en \\
\hline Estructural & $\begin{array}{l}\text { apoB (ApoB - } 48 \text { y } \\
\text { apoB - 100) }\end{array}$ & La columna vertebral de las lipoproteínas & Quilomicrones, VLDL, y LDL \\
\hline \multirow{4}{*}{$\begin{array}{l}\text { Cofactores/ } \\
\text { inhibidores } \\
\text { enzimáticos }\end{array}$} & ApoC-II & Coactivador de lipoproteína lipasa & HDL, quilomicrones y VLDL \\
\hline & ApoA-I & $\begin{array}{l}\text { Coenzima de lecitina: colesterol aciltransferasa } \\
\text { (LCAT) }\end{array}$ & $\begin{array}{l}\text { Principalmente HDL, también se } \\
\text { puede encontrar en quilomicrones }\end{array}$ \\
\hline & ApoC-I & $\begin{array}{l}\text { Inhibidor de la transferencia de proteína de } \\
\text { éster de colesterol (CETP) }\end{array}$ & $\begin{array}{l}\text { Principalmente HDL, visto en otras } \\
\text { lipoproteínas en estado posprandial }\end{array}$ \\
\hline & ApoA - II & Inhibidor de la lipoproteína lipasa & HDL \\
\hline \multirow[t]{2}{*}{ Ligandos } & ApoE & $\begin{array}{l}\text { Se une al receptor de } L D L \text { y a la proteína-1 } \\
\text { relacionada con el receptor de } L D L \text {, permitiendo } \\
\text { la eliminación de lipoproteínas de la circulación }\end{array}$ & $\mathrm{HDL}$, quilomicrones, VLDL y LDL \\
\hline & ApoC- III & $\begin{array}{l}\text { Antagonista ApoE, bloquea la eliminación de las } \\
\text { lipoproteínas circulantes }\end{array}$ & VLDL, LDL \\
\hline
\end{tabular}


y colesterol adicionales a través de efluentes celulares, así como por transferencia de componentes de superficie desde quilomicrones y VLDL durante la lipólisis intravascular $^{(28)}$. El receptor B1 (SR - B1) se ha identificado como un receptor HDL de doble función. En el hígado y en los tejidos esteroides, se une a HDL a través de apoA - I, y el éster de colesterol se entrega selectivamente a las células, aunque la partícula HDL en sí no se absorbe. En otros tejidos, SR - B1 media la aceptación del colesterol celular por parte de HDL, que esterifica el colesterol libre y lo transporta al hígado para su excreción biliar ${ }^{(29)}$. Las concentraciones plasmáticas de HDL muestran una fuerte relación inversa independiente con el riesgo de enfermedad cardiovascular aterosclerótica. Aunque el HDL tiene propiedades antioxidantes, antiinflamatorias, vasodilatadoras y antitrombóticas, es probable que la actividad central antiaterogénica del HDL sea su capacidad para eliminar el colesterol y los oxiesteroles de las células espumosas de macrófagos, las células de músculo liso y las células endoteliales de la pared arterial ${ }^{(30)}$.

Después de pasar por todo el transporte necesario para llegar a las células, la grasa llega a las mitocondrias en forma de ácidos grasos libres. Dentro de las mitocondrias, la descomposición de los ácidos grasos continúa en un proceso de cuatro pasos llamado el ciclo de oxidación $\beta$, un proceso reiterativo que elimina dos carbonos del ácido graso en cada ciclo ${ }^{(22)}$. Este proceso es inhibido por la insulina durante el período posprandial ${ }^{(31)}$. Como se ha mencionado anteriormente, un estudio de cicloergometría en voluntarios humanos ${ }^{(11)}$ mostró que la contribución relativa de la oxidación de la grasa al gasto energético total se reduce solo cuando la intensidad del ejercicio se acerca a $75 \%$ del $\dot{\mathrm{VO}}_{2 \text { máx }}$. Esto significa que la oxidación de la grasa se satura con AF de alta intensidad. Sin embargo, la capacidad de oxidación de la grasa puede ser mejorada por medio de $\mathrm{AF}$ de resistencia que induce en el tejido muscular: i. expresión aumentada de transportadores de ácidos grasos en la membrana plasmática, ii. mayor densidad de mitocondrias iii. proliferación de capilares, y iv. transporte más rápido de ácidos grasos del citosol a la mitocondria. Todos estos efectos, en conjunto, resultan en un mejor suministro de ácidos grasos y en una mayor oxidación ${ }^{(32)}$.

\section{Efecto cuantitativo de la práctica del ejercicio sobre los lípidos y las lipoproteínas plasmáticas}

Los efectos positivos de la actividad física y el ejercicio sobre la protección cardiovascular han sido amplia- mente validados. Cada aumento de 1 MET en el rendimiento de ejercicio confiere una reducción de la mortalidad total de cerca de $12 \%{ }^{(33)}$. Una proporción importante de este beneficio está mediada por el metabolismo de los lípidos, incluyendo impactos positivos sobre los triglicéridos plasmáticos, LDL, HDL Lp(a), e incluso apolipoproteínas como apoC - III.

\section{Impacto del ejercicio sobre los triglicéridos plasmáticos}

Los triglicéridos plasmáticos son uno de los componentes del perfil de lípidos más susceptibles de cambiar a través de modificaciones en el estilo de vida. En un amplio estudio epidemiológico realizado en Brasil, la práctica regular de al menos 150 minutos/ semana de actividad física vigorosa se asoció a niveles significativamente más altos de HDLc y más bajos de triglicéridos ${ }^{(34)}$. En el Health Professionals Follow-up Study (HPFS), un estudio de cohorte prospectivo de hombres estadounidenses, la diferencia en la relación TG/HDLc entre los quintiles superior e inferior de la práctica de actividad física fue $-26 \%{ }^{(35)}$.

En individuos con obesidad, un meta-análisis Cochrane que incluyó 43 estudios con 3.476 participantes encontró que una intervención de actividad física que duró al menos 12 semanas indujo una disminución media en la TG en ayunas de $18 \mathrm{mg} / \mathrm{dL}$, independiente de las modificaciones nutricionales ${ }^{(36)}$. Otro meta-análisis del entrenamiento de resistencia regular entre los hombres, que incluyó 49 estudios y 2.990 participantes, encontró una disminución media de TG de $9 \%$ y una correlación positiva entre la disminución de TG y la intensidad del ejercicio ${ }^{(37)}$.

En general, las pruebas actuales indican que el ejercicio y la actividad física tienen un impacto sobre los triglicéridos plasmáticos, y su efecto está en gran medida relacionado con la intensidad y la frecuencia de la práctica.

\section{Impacto del ejercicio en el plasma HDLc}

Algunos estudios se han centrado en el efecto del ejercicio y la actividad física y el ejercicio sobre el HDLc en niños. Un estudio transversal de 1.731 adolescentes de 12 a 19 años de edad analizó la actividad física medida con acelerómetro para encontrar la intensidad mínima que le conferiría beneficios a la salud. Después de ajustar los posibles factores de confusión, cada hora/día adicional de actividad de intensidad luminosa se aso- 
ció a $1,5 \mathrm{mg} / \mathrm{dL}\left(0,03\right.$ a 2,6) de HDLc en plasma ${ }^{(38)}$. Otro estudio de 3.984 jóvenes de 6 a 17 años de edad de la National Health and Nutrition Examination Survey (NHANES 2003 - 2006) encontró que por cada $1 \%$ adicional de tiempo en actividad física de moderada a vigorosa, los triglicéridos plasmáticos fueron menores en $2,08 \mathrm{mg} / \mathrm{dL}(-3,89 \mathrm{a}-0,27)$ y HDL-C fue mayor en $0,47 \mathrm{mg} / \mathrm{dL}(0,25 \text { a } 0,69)^{(39)}$.

El impacto del ejercicio sobre el HDL no se limita a las concentraciones de HDLc, sino también al tamaño de las lipoproteínas, que se ha demostrado cambian hacia partículas más grandes y presumiblemente más funcionales. El efecto cuantitativo del ejercicio aeróbico regular sobre el HDLc en plasma se ha estimado en un aumento de $10 \%-15 \%{ }^{(40)}$.

\section{Impacto del ejercicio en los niveles del plasmáticos de colesterol LDL}

La relación entre los niveles de actividad física y el LDLc es mucho menos pronunciada que para el HDLc o los triglicéridos. En un estudio prospectivo de 8 meses con 109 participantes, la actividad física vigorosa (hasta 75 minutos/semana) redujo de manera significativa los niveles de LDLc, pero la reducción numérica fue modesta (de $115 \pm 33,4$ a 109,8 $\pm 31,7 \mathrm{mg} / \mathrm{dL}, \mathrm{p}=$ $0,04)^{(41)}$. Otros estudios han demostrado un efecto aún menor: Ocho ensayos investigaron el efecto del ejercicio aeróbico sobre el LDLc, mostrando pruebas sólidas de heterogeneidad (I2 $=86 \%, \mathrm{p}<0,001$ ). Después de la compilación de los resultados mediante un modelo de efectos aleatorios en un meta-análisis, la estimación combinada del cambio de LDLc con ejercicio aeróbico no fue significativamente diferente de cero (diferencia de medias ponderada versus grupo control: - 0,52; IC de $95 \%$ : $-7,97$ a 6,92$)^{(42)}$.

Estos resultados confirman lo que se sabe sobre la fisiología de LDLc, es decir, que la producción de colesterol hepático tiene una gran influencia genética, está modestamente influenciada por la dieta y muy poco por el ejercicio.

\section{Impacto del ejercicio sobre el colesterol no HDL}

El colesterol no HDL o colesterol aterogénico (es decir, la suma de colesterol VLDL, colesterol LDL, colesterol lipoproteico remanente y colesterol $\mathrm{Lp}(\mathrm{a})$ ), se considera un factor de riesgo cardiovascular alto y consistente ${ }^{(43)}$. La mayoría de las pruebas que evalúan específicamente la asociación entre la actividad física y el colesterol no HDL provienen de estudios en pobla- ciones jóvenes. Un estudio que dio seguimiento a 108 adolescentes que tenían cirugía bariátrica por 3 años correlacionó una cantidad mayor de actividad física con mayor disminución absoluta en el no-HDL-C, a pesar de los bajos conteos absolutos de pasos y la lentitud de la cadencia ${ }^{(4)}$. Un estudio de cohorte que incluyó a 880 escolares noruegos con una edad media de 10,2 años demostró que la sustitución de 30 minutos diarios de sedentarismo por 30 minutos de actividad física moderada a vigorosa (> 2.296 recuentos de acelerómetros/ min) se asoció inversamente con la concentración de colesterol no $\mathrm{HDL}^{(45)}$.

\section{Impacto del ejercicio sobre la apolipoproteína C - III en plasma}

La ApoC - III ha sido identificada como un factor de riesgo cardiovascular independiente debido a su antagonismo con el receptor LDL y al obstáculo de las funciones protectoras del $\mathrm{HDL}^{(46-48)}$. En un estudio transversal con 3.631 participantes, cada 20 horas MET adicionales por semana de actividad física se asociaron a concentraciones $0,9(-1,7$ a - 0,1) inferiores de HDL que contenían apoC - III ${ }^{(49)}$.

\section{Impacto del ejercicio en las lipoproteínas plasmáticas(a)}

Varios estudios de aleatorización de grandes cohortes y mendelianos han demostrado que la lipoproteína(a) en plasma $\mathrm{Lp}(\mathrm{a})$, presenta una asociación negativa y al menos parcialmente causal con el riesgo cardiovascular $^{(50)}$. Una asociación negativa entre la $\mathrm{Lp}(\mathrm{a})$ y la actividad física parece ser en particular sorprendente entre los niños: un gran estudio de seguimiento multicéntrico de 2.464 niños y adultos jóvenes (de 9 a 24 años de edad) encontró que la Lp(a) plasmática se correlacionaba en forma significativa solo con el nivel de actividad física, pero no con la edad, el sexo $\mathrm{u}$ otros factores de riesgo cardiovascular conocidos. Un estudio transversal similar en 1.340 adultos correlacionó los niveles de actividad física en el IPAQ (Cuestionario Internacional de Actividad Física) con el $L p(a)$ plasmático. Las concentraciones de $\mathrm{Lp}(\mathrm{a})$ en participantes con niveles de actividad física bajos, moderados y altos fueron respectivamente 29,2 \pm $13,7,26,3 \pm 12,9$ y $24,5 \pm 11,4 \mathrm{mg} / \mathrm{dL}$ con diferencias significativas entre grupos ${ }^{(51)}$. Estos resultados sugieren que, a diferencia de LDLc, la Lp(a) tiende a responder al ejercicio y a la actividad física de una manera que responde a la dosis. 


\section{Efectos diferenciales de las modalidades de ejercicio sobre los lípidos plasmáticos}

En general, los efectos de la actividad física sobre la salud dependen de su volumen (producto de la intensidad del ejercicio y del tiempo de ejercicio), de la frecuencia (cantidad de unidades de entrenamiento por semana o mes) y de su modalidad (aeróbica, de resistencia o combinada) ${ }^{(52)}$.

\section{EJERCICIO DE RESISTENCIA (ENDURANCE)}

Tanto el ejercicio aeróbico intermitente (períodos cortos a altas intensidades) como el ejercicio aeróbico continuo disminuyen el colesterol total y aumentan el colesterol HDL en sujetos sedentarios ${ }^{(53)}$. Se ha estimado que el umbral para el aumento del HDLc inducido por el ejercicio aeróbico es de unas $1.000 \mathrm{kcal}$ de carrera o un ejercicio aeróbico similar por semana ${ }^{(54)}$. En un meta-análisis de 51 intervenciones que incluyeron a 4.700 pacientes que se sometieron a programas de ejercicio aeróbico durante al menos 12 semanas, HDLc, TG y LDLc cambiaron en promedio + 4,6 \% - 3,7 \% y$5 \%$, respectivamente, sin cambios en el colesterol total. El HDLc fue el componente del perfil lipídico más propenso a cambiar con el ejercicio aeróbico, incluso un programa de 10 semanas (tres veces a la semana a $85 \%$ de la frecuencia cardíaca máxima) indujo un aumento de $13 \%$ en el HDLc. El ejercicio aeróbico prolongado (150 $\mathrm{min} / \mathrm{semana}$ ) fue superior para mejorar los parámetros de lípidos en comparación con un protocolo de intervalo intenso (40 min/semana).

\section{EJERCICIO DE RESISTENCIA (RE)}

Los resultados sobre el efecto del ejercicio de resistencia en los lípidos sanguíneos han sido más contradictorios que en el ejercicio aeróbico ${ }^{(52)}$. Un estudio reciente en mujeres adultas encontró un impacto notable de solo 12 semanas de ejercicio de resistencia usando el propio peso corporal del participante, reduciendo el TG en 14,3\% mientras aumentó el HDLc en 7,9 \% ${ }^{(55)}$. Un pequeño estudio separado, restringido a mujeres posmenopáusicas, informó también reducciones significativas en el LDLc con 150 minutos/semana de ejercicio de resistencia ${ }^{(56)}$. En los hombres, dos pequeños estudios han examinado de manera específica el impacto del ejercicio de resistencia en los lípidos plasmáticos. En uno de ellos, el efecto agudo (72-h) de $75 \%$ de las repeticiones máximas sobre el TG plasmático se estimó en - 11,0 mg/dL ${ }^{(57)}$. En un segundo estudio, los pacientes que participaron en un programa de ejercicios de resistencia moderada ( $45 \%$ - $55 \%$ de las repeticiones máximas) o de alta intensidad ( $80 \%$ - $90 \%$ de las repeticiones máximas) experimentaron reducciones significativas de LDLc (- 12,2 a - 13,5 mg/dL). El grupo de alta intensidad también experimentó aumentos significativos de $\operatorname{HDLc}(+5,5 \mathrm{mg} / \mathrm{dL})^{(58)}$.

\section{MODALIDADES COMBINADAS}

Se espera que la combinación de ejercicios aeróbicos y de resistencia proporcione los beneficios acumulativos de ambas modalidades, pero los datos sobre los protocolos de intervención combinados han mostrado efectos inconsistentes sobre los lípidos plasmáticos ${ }^{(59)}$. En general, el impacto de las intervenciones que combinan ambas modalidades es comparable al obtenido con el ejercicio aeróbico, en todas las subfracciones lipídicas importantes ${ }^{(60)}$.

\section{Prescripción de ejercicios en pacientes con dislipidemia}

Las recomendaciones de grado A basadas en la evidencia sobre la actividad física en pacientes con dislipidemia pueden resumirse de la siguiente manera ${ }^{(61)}$ :

- Los objetivos de LDLc dependen del estado de riesgo cardiovascular global (GCVR) del paciente ${ }^{(62)}$ :

- $130 \mathrm{mg} / \mathrm{dL}$ para pacientes de bajo riesgo (sin eventos cardiovasculares previos, sin factores de riesgo cardiovascular y GCVR estimado < $10 \%$ )

- $100 \mathrm{mg} / \mathrm{dL}$ para pacientes de riesgo moderado o alto (sin eventos cardiovasculares previos, 1 - 2 factores de riesgo cardiovascular y GCVR estimada $10 \%$ - $20 \%$, o diabetes o enfermedad renal crónica sin factores de riesgo adicionales).

- $70 \mathrm{mg} / \mathrm{dL}$ para pacientes de muy alto riesgo (evento cardiovascular previo, o GCVR estimado $>=20 \%$, o hipercolesterolemia familiar, o diabetes o enfermedad renal crónica más uno o más factores de riesgo no controlados).

- $55 \mathrm{mg} / \mathrm{dL}$ para pacientes de riesgo extremo (evento cardiovascular previo + diabetes o enfermedad renal crónica, o enfermedad cardiovascular progresiva a pesar de LDLc por debajo de $70 \mathrm{mg} / \mathrm{dL}$, o enfermedad cardiovascular antes de los 55 años en hombres o antes de los 65 años en mujeres).

- Las sesiones de ejercicio deben durar $>40$ minutos, el programa de entrenamiento debe durar $>40$ semanas.

- Si es posible, es deseable realizar ejercicios de resistencia de al menos cinco grupos de músculos gran- 
des, como 8 - 10 repeticiones, cada serie a $70 \%$ - $85 \%$ de las repeticiones máximas.

- El ejercicio en pacientes dislipidémicos debe prescribirse junto con una farmacoterapia adecuada para reducir los lípidos y modificaciones dietéticas.

Las recomendaciones se pueden ajustar a la condición general del paciente; estas recomendaciones se resumen en la Tabla $2^{(59)}$.

\section{CONCLUSIÓN}

La actividad física y el ejercicio tienen el potencial de tener un impacto positivo en los lípidos plasmáticos, en especial los triglicéridos, el colesterol HDL y, en menor medida, el colesterol LDL, el Lp(a) y probablemente el apoC-III. Sin embargo, el impacto general del ejercicio sobre el riesgo cardiovascular va más allá de su impacto sobre los lípidos plasmáticos.

\section{Conflicto de intereses}

Los autores no manifiestan ningún conflicto de intereses que comunicar.

\section{Financiación}

Los autores no recibieron financiación específica para la redacción de este artículo.

\section{Declaración de autoría}

EASN, DRM, COM participaron en la concepción y la redacción del artículo. Todos los autores revisaron de artículo y validaron su versión final.

Tabla 2. Resumen de recomendaciones para la práctica de ejercicios en pacientes con dislipidemia según la actividad (movilidad) general. Adaptado de ${ }^{(59)}$

\begin{tabular}{|l|l|l|}
\hline \multicolumn{1}{|c|}{ Estatus del paciente } & \multicolumn{1}{|c|}{ Objetivos } & \multicolumn{1}{c|}{ Recomendaciones de actividad física } \\
\hline Móvil, sin dislipidemia & $\begin{array}{l}\text { Mantener bajos los niveles } \\
\text { de LDLc y TG, aumentar } \\
\text { el HDLc }\end{array}$ & $\begin{array}{l}>30 \text { min/día, } 5 \text { veces/semana de ejercicio aeróbico a } 70 \%-80 \% \text { de la } \\
\text { reserva de frecuencia cardíaca, combinado con ejercicio de resistencia a } \\
50 \% \text { de las repeticiones máximas. }\end{array}$ \\
\hline Móvil, con dislipidemia & $\begin{array}{l}\text { Reducir LDLc y TG, } \\
\text { aumentar HDLc }\end{array}$ & $\begin{array}{l}>30 \text { min/día, } 5 \text { veces/semana de ejercicio aeróbico a } 70 \%-80 \% \text { de la } \\
\text { reserva de frecuencia cardíaca, progresando a } 85 \% \text { de la reserva de } \\
\text { frecuencia cardíaca, combinado con ejercicio de resistencia a } 75 \% \text { - } 85 \% \\
\text { de las repeticiones máximas. }\end{array}$ \\
\hline $\begin{array}{l}\text { Movilidad limitada } \\
\text { (discapacitados, ancianos), } \\
\text { con dislipidemia }\end{array}$ & $\begin{array}{l}\text { Reducir LDLc y TG, } \\
\text { aumentar HDLc }\end{array}$ & $\begin{array}{l}\text { Aumentar la actividad física en la medida de lo posible, progresando en } \\
\text { el ejercicio de resistencia de } 50 \% \text { a } 75 \% \text { de las repeticiones máximas en } \\
\text { los principales grupos musculares. }\end{array}$ \\
\hline
\end{tabular}

\section{Referencias bibliográficas}

1. Hawley JA, Hargreaves M, Joyner MJ, Zierath JR. Integrative Biology of Exercise. Cell. 2014;159(4):738-49.

2. Tremblay MS, Colley RC, Saunders TJ, Healy GN, Owen N. Physiological and health implications of a sedentary lifestyle. Appl Physiol Nutr Metab. 2010;35(6):725-40.

3. Organización Mundial de la Salud. Actividad física [online]. 2019. [Accessed 4 Jun. 2019]. Available at: https://www.who. int/dietphysicalactivity/pa/es/

4. Caspersen CJ, Powell KE, Christenson GM. Physical activity, exercise, and physical fitness: definitions and distinctions for health-related research. Public Health Rep. 1985; 100(2): 126-31.
5. Ferguson B. ACSM's Guidelines for Exercise Testing and Prescription 9th Ed. 2014. J Can Chiropr Assoc. 2014;58(3):328.

6. Ortega FB, Ruiz JR, Castillo MJ, Sjöström M. Physical fitness in childhood and adolescence: a powerful marker of health. Int J Obes (Lond). 2007; 32(1): 1-11.

7. Mohrman DE, Heller LJ. Cardiovascular physiology. 9th edition. New York: McGraw-Hill; 2018.

8. Dempsey JA, Blain GM, Amann M. Are type III-IV muscle afferents required for a normal steady-state exercise hyperpnoea in humans? J Physiol. 2014;592(3):463-74.

9. Ball D. Metabolic and endocrine response to exercise: sympathoadrenal integration with skeletal muscle. J Endocrinol. 2015;224(2):R79-95. 
10. Havel RJ, Naimark A, Borchgrevink CF. Turnover rate and oxidation of free fatty acids of blood plasma in man during exercise: studies during continuous infusion of palmitate1-C14. J Clin Invest. 1963; 42(7):1054-63.

11. Van Loon LJ, Greenhaff PL, Constantin-Teodosiu D, Saris $\mathrm{WH}$, Wagenmakers AJ. The effects of increasing exercise intensity on muscle fuel utilisation in humans. J Physiol. 2001; 536(Pt1): 295-304.

12. Scott W, Stevens J, Binder-Macleod SA. Human skeletal muscle fiber type Classifications. Phys Ther. 2001;81(11): 1810-6.

13. Peter JB, Barnard RJ, Edgerton VR, Gillespie CA, Stempel KE. Metabolic profiles of three fiber types of skeletal muscle in guinea pigs and rabbits. Biochemistry. 1972;11(14):262-33.

14. Hardie DG. AMP-activated protein kinase: A key system mediating metabolic responses to exercise. Med Sci Sports Exerc. 2004; 36(1): 28-34.

15. Jäger S, Handschin C, St-Pierre J, Spiegelman BM. AMPactivated protein kinase (AMPK) action in skeletal muscle via direct phosphorylation of PGC-1alpha. Proc Natl Acad Sci USA. 2007;104(29):12017-22.

16. Lin J, Handschin C, Spiegelman BM. Metabolic control through the PGC-1 family of transcription coactivators. Cell Metab. 2005;1(6):361-70.

17. Wideman L, Weltman JY, Hartman ML, Veldhuis JD, Weltman A. Growth hormone release during acute and chronic aerobic and resistance exercise. Sports Med. 2002; 32(15):987-1004.

18. Perrini S, Laviola L, Carreira MC, Cignarelli A, Natalicchio A, Giorgino F. The GH/IGF1 axis and signaling pathways in the muscle and bone: mechanisms underlying age-related skeletal muscle wasting and osteoporosis. J Endocrinol. 2010; 205(3): 201-10.

19. Bodine S, Stitt TN, Gonzalez M, Kline WO, Stover GL, Bauerlein $\mathrm{R}$, et al. Akt/mTOR pathway is a crucial regulator of skeletal muscle hypertrophy and can prevent muscle atrophy in vivo. Nat Cell Biol. 2001; 3(11): 1014-9.

20. Liu Z, Jahn LA, Wei L, Long W, Barrett EJ. Amino acids stimulate translation initiation and protein synthesis through an Akt-independent pathway in human skeletal muscle. J Clin Endocrinol Metab. 2002; 87(12): 5553-8.

21. Léger B, Cartoni R, Praz M, Lamon S, Dériaz O, Crettenand A, et al. Akt signalling through GSK-3beta, mTOR and Foxo 1 is involved in human skeletal muscle hypertrophy and atrophy. J Physiol. 2006; 576(Pt 3): 923-33.

22. Janson LW, Tischler ME. The Big Picture: Medical Biochemistry. New York: McGraw-Hill; 2018.

23. Nikkari T, Luukkainen P, Pietinen P, Puska P. Fatty acid composition of serum lipid fractions in relation to gender and quality of dietary Fat. Ann Med. 1995; 27(4): 491-8.

24. Kwiterovich PO Jr. The metabolic pathways of high-density lipoprotein, low-density lipoprotein, and triglycerides: a current review. Am J Cardiol. 2000; 86(12A): 5L-10L.
25. Redgrave TG. Chylomicron metabolism. Biochem Soc Trans. 2004; 32 (Pt 1): 79-82.

26. Cladaras C, Hadzopoulou-Cladaras M, Nolte RT, Atkinson D, Zannis VI. The complete sequence and structural analysis of human apolipoprotein B-100: relationship between apoB-100 and apoB-48 forms. EMBO J. 1986; 5(13): 3495-507.

27. Vaisar T. Proteomics investigations of HDL: challenges and promise. Curr Vasc Pharmacol. 2012; 10(4): 410-21.

28. Lewis GF. Determinants of plasma HDL concentrations and reverse cholesterol transport. Curr Opin Cardiol. 2006; 21(4): 345-52.

29. Bender DA, Botham KM, Kennelly PJ, Rodwell VW, Weil PA, et al. Harper's Illustrated Biochemistry. 31st edition. New York: McGraw-Hill; 2018.

30. Tall AR. Cholesterol efflux pathways and other potential mechanisms involved in the athero-protective effect of high density lipoproteins. J Intern Med. 2008; 263(3): 256-73.

31. Coyle EF, Jeukendrup AE, Wagenmakers AJ, Saris WH. Fatty acid oxidation is directly regulated by carbohydrate metabolism during exercise. Am J Physiol. 1997; 273(2 Pt 1): E26875.

32. Horowitz JF, Klein S. Lipid metabolism during endurance exercise. Am J Clin Nutr. 2000; 72(2 Suppl): 558S-63S.

33. Ruegsegger GN, Booth FW. Health Benefits of Exercise. Cold Spring Harb Perspect Med. 2018; 8(7): pii: a029694.

34. Silva RC, Diniz Mde F, Alvim S, Vidigal PG, Fedeli LM, Barreto SM. Physical Activity and Lipid Profile in the ELSABrasil Study. Arq Bras Cardiol. 2016;107(1):10-9.

35. Lee DH, de Rezende LFM, Eluf-Neto J, Wu K, Tabung FK, Giovannucci EL. Association of type and intensity of physical activity with plasma biomarkers of inflammation and insulin response. Int J Cancer. 2019;145(2):360-9.

36. Shaw K, Gennat H, O’Rourke P, Del Mar C. Exercise for overweight or obesity. Cochrane Database Syst Rev. 2006; (4):CD003817.

37. Fikenzer K, Fikenzer S, Laufs U, Werner C. Effects of endurance training on serum lipids. Vascul Pharmacol. 2018;101: 9-20.

38. Carson V, Ridgers ND, Howard BJ, Winkler EA, Healy GN, Owen N, et al. Light-intensity physical activity and cardiometabolic biomarkers in US adolescents. PLoS One. 2013; 8(8): e71417.

39. Jenkins GP, Evenson KR, Herring AH, Hales D, Stevens J. Cardiometabolic Correlates of Physical Activity and Sedentary Patterns in U.S. Youth. Med Sci Sports Exerc. 2017;49(9): 1826-33.

40. Woudberg NJ, Mendham AE, Katz AA, Goedecke JH, Lecour S. Exercise intervention alters HDL subclass distribution and function in obese women. Lipids Health Dis. 2018; 17(1):232.

41. Sponder M, Campean IA, Dalos D, Emich M, Fritzer-Szekeres M, Litschauer B, et al. Effect of long-term physical activity on PCSK9, high- and low-density lipoprotein cholesterol, and 
lipoprotein(a) levels: a prospective observational trial. Pol Arch Intern Med. 2017;127(7-8):506-11.

42. Cai M, Zou Z. Effect of aerobic exercise on blood lipid and glucose in obese or overweight adults: A meta-analysis of randomized controlled trials. Obes Res Clin Pract. 2016;10(5):589602.

43. Millán J, Hernández-Mijares A, Ascaso JF, Blasco M, Brea A, Díaz Á, et al. The real measurement of non-HDL-cholesterol: Atherogenic cholesterol. Clin Investig Arterioscler. 2016; 28(6): 265-70.

44. Price PH, Kaizer AM, Daniels SR, Jenkins TM, Inge TH, Eckel RH. Phisical Activity Improves Lipid and WeightLoss Outcomes After Metabolic Bariatric Surgery in Adolescents with Severe Obesity. Obesity (Silver Spring). 2019;27(6):989-96.

45. Jones PR, Rajalahti T, Resaland GK, Aadland E, SteeneJohannessen J, Anderssen SA, et al. Associations of PA and sedentary time with lipoprotein subclasses in Norwegian schoolchildren: The Active Smarter Kids (ASK) study. Atherosclerosis. 2019. pii: S0021-9150(19)30447-2.

46. Mendivil CO, Zheng C, Furtado J, Lel J, Sacks FM. Metabolism of very-low-density lipoprotein and low-density lipoprotein containing apolipoprotein C-III and not other small apolipoproteins. Arterioscler Thromb Vasc Biol. 2010;30(2):239-45.

47. Mendivil CO, Rimm EB, Furtado J, Chiuve SE, Sacks FM. Low-density lipoproteins containing apolipoprotein C-III and the risk of coronary heart disease. Circulation. 2011; 124(19):2065-72.

48. Jensen MK, Rimm EB, Furtado JD, Sacks FM. Apolipoprotein C-III as a Potential Modulator of the Association Between HDL-Cholesterol and Incident Coronary Heart Disease. J Am Heart Assoc. 2012; 1(2): pii: jah3-e000232.

49. Koch M, Furtado JD, Jiang GZ, Gray BE, Cai T, Sacks F, et al. Associations of anthropometry and lifestyle factors with HDL subspecies according to apolipoprotein C-III. J Lipid Res. 2017; 58(6):1196-203.

50. Wilson DP, Jacobson TA, Jones PH, Koschinsky ML, McNeal CJ, Nordestgaard BG, et al. Use of Lipoprotein(a) in clinical practice: A biomarker whose time has come. A scientific statement from the National Lipid Association. J Clin Lipidol. 2019; 13(3):374-92.

51. Bermúdez V, Aparicio D, Rojas E, Peñaranda L, Finol F, Acosta $\mathrm{L}$, et al. An elevated level of physical activity is associated with normal lipoprotein(a) levels in individuals from Maracaibo, Venezuela. Am J Ther. 2010;17(3):341-50.

52. Vanhees L, Geladas N, Hansen D, Kouidi E, Niebauer J, Reiner $\mathrm{Z}$, et al. Importance of characteristics and modalities of physi- cal activity and exercise in the management of cardiovascular health in individuals with cardiovascular risk factors: recommendations from the EACPR. Part II. Eur J Prev Cardiol. 2012;19(5):1005-33.

53. Altena TS, Michaelson JL, Ball SD, Guilford BL, Thomas TR. Lipoprotein subfraction changes after continuous or intermittent exercise training. Med Sci Sports Exerc. 2006; 38(2):36772.

54. Wood PD, Haskell WL, Blair SN, Williams PT, Krauss RM, Lindgren FT, et al. Increased exercise level plasma lipoprotein concentrations: a one-year, randomized, controlled study in sedentary middle-aged men. Metabolism. 1983;32(1):31-9.

55. Zapata-Lamana R, Cigarroa I, Diaz E, Saavedra C. Resistance exercise improves serum lipids in adult women. Rev Med Chil. 2015;143(3):289-96.

56. Prabhakaran B, Dowling EA, Branch JD, Swain DP, Leutholtz BC. Effect of 14 weeks of resistance training on lipid profile and body fat percentage in premenopausal women. Br J Sports Med. 1999;33(3):190-5.

57. Lira FS, Yamashita AS, Uchida MC, Zanchi NE, Gualano B, Martins E Jr, et al. Low and moderate, rather than high intensity strength exercise induces benefit regarding plasma lipid profile. Diabetol Metab Syndr. 2010;2:31.

58. Sheikholeslami Vatani D, Ahmadi S, Ahmadi Dehrashid K, Gharibi F. Changes in cardiovascular risk factors and inflammatory markers of young, healthy, men after six weeks of moderate or high intensity resistance training. J Sports Med Phys Fitness. 2011; 51(4):695-700.

59. Mann S, Beedie C, Jimenez A. Differential effects of aerobic exercise, resistance training and combined exercise modalities on cholesterol and the lipid profile: review, synthesis and recommendations. Sports Med. 2014; 44(2):211-21.

60. Shaw I, Shaw BS, Krasilshchikov O. Comparison of aerobic and combined aerobic and resistance training on low-density lipoprotein cholesterol concentrations in men. Cardiovasc J Afr. 2009;20(5):290-5.

61. Hansen D, Niebauer J, Cornelissen V, Barna O, Neunhäuserer D, Stettler C, et al. Exercise Prescription in Patients with Different Combinations of Cardiovascular Disease Risk Factors: A Consensus Statement from the EXPERT Working Group. Sports Med. 2018; 48(8):1781-97.

62. Jellinger PS, Handelsman Y, Rosenblit PD, Bloomgarden ZT, Fonseca VA, Garber AJ, et al. American Association of Clinical Endocrinologists and American College of Endocrinology Guidelines for management of dyslipidemia and prevention of cardiovascular disease. Endocr Pract. 2017;23(Suppl 2):1-87. 